[10] Note that the $\pi$-system of fulvenyl anion 1 as well as of fulvenyl radical 2 is considerably delocalized according to HMO calculations.

[11] J. Hine, D.B. Knight, J. Org. Chem. 1970 , 35, 3946; D.B. Knight, R.L. Hartless, D.A. Jarvis, ibid. 1972, 37, 688 .

[12] Oxidative coupling may be realized by adding anh. $\mathrm{CuCl}_{2}$ to the cooled $\left(-78^{\circ}\right)$ solution of anion 1 as well. While product distribution is similar to that of Table $l$, the total anal. yield (determined by 1 H-NMR) drops to $68 \%$.

[13] Spectroscopic structure elucidation of compounds 3-7 will be discussed in a full paper (in preparation for Helv. Chim. Acta). Spectra of purified compounds are given in [1]

[14] Tautomerizations of products with cyclopentadiene units (see 5-7) may occur at low temperature by base catalysis or, in many cases, at ambient temperature, by concerted 1,5-H shifts [9].
[15] While HPLC allowed to separate tautomers $5 \mathbf{a}$ and $5 \mathbf{b}$, the mixture of tautomers 6 could not be separated.

[16] Coupling at C(6) of 2 would produce a diradical and is, therefore, considered to be energetically very unfavorable.

[17] An adequate portion of this oil was used for NMR analysis at $300 \mathrm{MHz}$ after adding a balanced amount of $\mathrm{MeNO}_{2}$ as an internal standard for integration.

\section{Synthese und Röntgenstruktur einer Triafulvalen-Vorstufe [1]}

\author{
Peter Engel, Christoph Läng [2], Michel Mühlebach und Markus \\ Neuenschwander*
}

Abstract. Triafulvalene precursors $\mathbf{3 b}$ and $\mathbf{3 c}$ have been synthesized by $\mathrm{Cu}^{\mathrm{II}}$-catalyzed 'carbene dimerization' of cyclopropyl carbenoids (obtained by halogen-lithium exchange of 1,1-dibromo-2-(phenylthio)-3-(trimethylsilyl)cyclopropane ( $\mathbf{2 b}$; see Scheme 2) in moderate yields. Diastereoselectivity of the central step $\mathbf{2 b} \rightarrow \mathbf{3 b}$ is low. An X-ray analysis of the predominantly formed trans-anti isomer has been performed.

\section{Einleitung}

Triafulvalen (Bi(cyclopropenyliden); 1) ist als gespanntes cyklisch gekreuzt konjugiertes System sowohl bindungstheoretisch wie spektroskopisch von grossem Interesse. Bis heute sind weder der Grundkörper 1, noch einfache Derivate, oder aussichtsreiche Vorstufen von 1 bekannt. Wir berichteten kürzlich von einer präparativ sehr einfachen Umsetzung von 1,1-Dibromo-2-(phenylthio)cyclopropan (2a) zum Diastereoisomerengemisch der Bi(cyclopropylidene) 3a [3]. Dabei wurde das unter kinetischer Kontrolle aus 2a mit BuLi erzeugte Gemisch der 1-Bromo-1lithio-2-(phenylthio)cyclopropane mittels $\mathrm{CuCl}_{2}$ in einer formalen 'Carben-Dimerisierung' zu 3a umgesetzt. Die präparativen Ausbeuten der Reaktion 2a $\rightarrow$ 3a sind sehr stark von den Reaktionsbedingungen abhängig. Das Resultat ist deshalb überraschend, weil 'Hückel-Anionen' wie Cy-

*Korrespondenz: Prof. Dr. M. Neuenschwander Institut für organische Chemie

Universität Bern

Freiestrasse 3

CH-3012 Bern clopentadienid unter analogen Bedingungen oxidative Kupplungen zu 1,1'-Bi(cyclopentadienylen) eingehen [4][5].

Mit dieser einfachen Eintopf-Reaktion $\mathbf{2 a} \rightarrow \mathbf{3 a}$ bietet sich erstmals die Möglichkeit, aussichtsreiche Vorstufen des Grundkörpers Triafulvalen (1) zu synthetisieren (Schema 1). Einerseits könnte versucht werden, die PhS-Gruppen von 3a in gute Abgangsgruppen umzuwandeln, was nach unseren früheren Erfahrungen mit analogen Triafulven-Vorstufen nicht so einfach ist [6]. Andererseits wäre die Einführung zweier sich bei der geplanten Elimination unterstützender Abgangsgruppen attraktiv. Eine kürzlich erschienene

Schema I

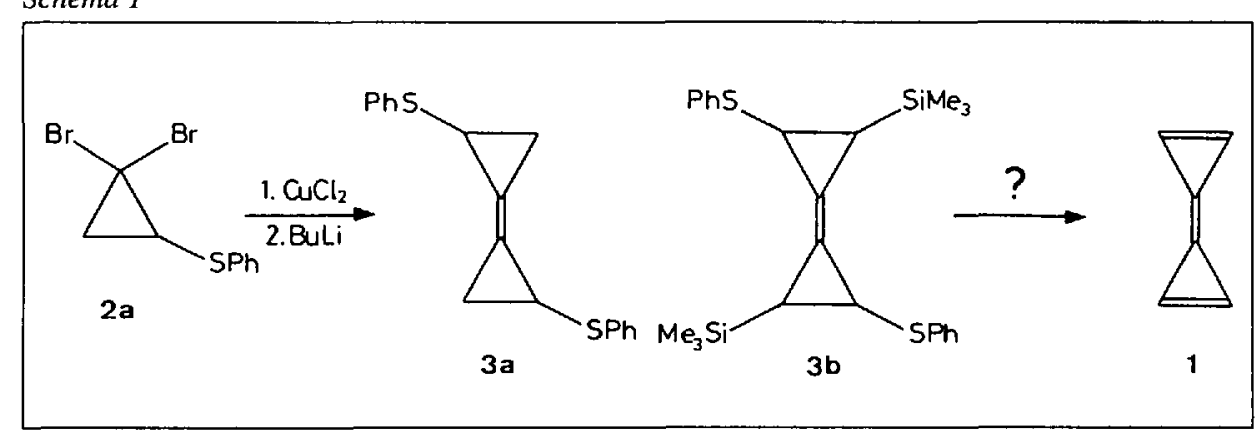

Arbeit über die Synthese des instabilen Spiro-pentadiens [7] veranlasst uns, im folgenden kurz über die erfolgreiche Synthese von Bi(cyclopropylidenen) des Typs 3b zu berichten.

\section{Synthese der Triafulvalen-Vorstufen $3 b$ und $3 c$}

trans-1,1-Dibromo-2-(phenylthio)-3(trimethylsilyl)cyclopropan (2b) ist weder nach Makosza [8] noch nach von Doering und Hoffmann [9] zugänglich und liess sich schliesslich aus dem entsprechenden Olefin nach Seyferth und Mitarbeitern [10] mittels (Tribromomethyl)(phenyl)quecksilber herstellen. Wird 2b zunächst bei $-95^{\circ}$ mit BuLi und dann mit EtOH behandelt, wird unter (Br-Li)Austausch in uiber $70 \%$ Ausbeute das erwartete Gemisch zweier diastereoisomerer trans-1-Bromo-2-(phenylthio)-3-(trimethylsilyl)cyclopropane isoliert [11]. Trotzdem ist die nachfolgende 'CarbenDimerisierung', welche durch $\mathrm{CuCl}_{2} \mathrm{ka}$ talysiert wird [3], sehr problematisch: Unter 'thermodynamischer Kontrolle' [12] liegen die maximalen Ausbeuten an $3 \mathrm{~b}$ bei $2 \%$. Sie steigen unter 'kinetischer Kontrolle' [12] auf 17\%, falls katalytische Mengen (rund 0,1 mol-equiv.) $\mathrm{CuCl}_{2}$ verwendet werden, und auf $21 \%$ (GC), falls 1 mol-equiv. $\mathrm{CuCl}_{2}$ zugegeben wird. $\mathrm{Im}$ Gegensatz zu 2a $\rightarrow$ 3a sinkt die Ausbeute der Umsetzung $\mathbf{2 b} \rightarrow \mathbf{3 b}$, wenn anstelle von THF als Lösungsmittel $\mathrm{Et}_{2} \mathrm{O}$ verwendet wird. Sonst sind die Ausbeuten in beiden Fällen von denselben Parametern abhängig (Details vgl. [3]), wobei vor 
[10] Note that the $\pi$-system of fulvenyl anion 1 as well as of fulvenyl radical $\mathbf{2}$ is considerably delocalized according to HMO calculations.

[11] J. Hine, D.B. Knight, J. Org. Chem. 1970, 35, 3946; D.B. Knight, R.L. Hartless, D.A. Jarvis, ibid. 1972, 37, 688.

[12] Oxidative coupling may be realized by adding anh. $\mathrm{CuCl}_{2}$ to the cooled $\left(-78^{\circ}\right)$ solution of anion 1 as well. While product distribution is similar to that of Table 1 , the total anal. yield (determined by lH-NMR) drops to $68 \%$.

[13] Spectroscopic structure elucidation of compounds 3-7 will be discussed in a full paper (in preparation for Helv. Chim. Acta).Spectra of purified compounds are given in [1].

[14] Tautomerizations of products with cyclopentadiene units (see 5-7) may occur at low temperature by base catalysis or, in many cases, at ambient temperature, by concerted $1,5-\mathrm{H}$ shifts [9].
[15] While HPLC allowed to separate tautomers $5 \mathbf{a}$ and $5 \mathbf{b}$, the mixture of tautomers 6 could not be separated.

[16] Coupling at C(6) of 2 would produce a diradical and is, therefore, considered to be energetically very unfavorable.

[17] An adequate portion of this oil was used for NMR analysis at $300 \mathrm{MHz}$ after adding a balanced amount of $\mathrm{MeNO}_{2}$ as an internal standard for integration.
Chimia 46 (1992) 380-382

(C) Neue Schweizerische Chemische Gesellschaft ISSN $0009-4293$

\title{
Synthese und Röntgenstruktur einer Triafulvalen-Vorstufe [1]
}

\author{
Peter Engel, Christoph Läng [2], Michel Mühlebach und Markus \\ Neuenschwander*
}

Abstract. Triafulvalene precursors $\mathbf{3 b}$ and $\mathbf{3 c}$ have been synthesized by $\mathrm{Cu}^{\mathrm{II}}$-catalyzed 'carbene dimerization' of cyclopropyl carbenoids (obtained by halogen-lithium exchange of 1,1-dibromo-2-(phenylthio)-3-(trimethylsilyl)cyclopropane (2b; see Scheme 2) in moderate yields. Diastereoselectivity of the central step $\mathbf{2 b} \rightarrow \mathbf{3 b}$ is low. An X-ray analysis of the predominantly formed trans-anti isomer has been performed.

\section{Einleitung}

Triafulvalen (Bi(cyclopropenyliden); 1) ist als gespanntes cyklisch gekreuzt konjugiertes System sowohl bindungstheoretisch wie spektroskopisch von grossem Interesse. Bis heute sind weder der Grundkörper 1, noch einfache Derivate, oder aussichtsreiche Vorstufen von 1 bekannt. Wir berichteten kürzlich von einer präparativ sehr einfachen Umsetzung von 1,1-Dibromo-2-(phenylthio)cyclopropan (2a) zum Diastereoisomerengemisch der Bi(cyclopropylidene) 3a [3]. Dabei wurde das unter kinetischer Kontrolle aus 2a mit BuLi erzeugte Gemisch der 1-Bromo-1lithio-2-(phenylthio)cyclopropane mittels $\mathrm{CuCl}_{2}$ in einer formalen 'Carben-Dimerisierung' zu 3a umgesetzt. Die präparativen Ausbeuten der Reaktion $\mathbf{2 a} \rightarrow 3 \mathbf{3}$ sind sehr stark von den Reaktionsbedingungen abhängig. Das Resultat ist deshalb überraschend, weil 'Hückel-Anionen' wie Cy-

\footnotetext{
*Korrespondenz: Prof. Dr. M. Neuenschwander Institut für organische Chemie

Universität Bern

Freiestrasse 3

CH-3012 Bern
}

clopentadienid unter analogen Bedingungen oxidative Kupplungen zu 1,1'-Bi(cyclopentadienylen) eingehen [4][5].

Mit dieser einfachen Eintopf-Reaktion 2a $\rightarrow$ 3a bietet sich erstmals die Möglichkeit, aussichtsreiche Vorstufen des Grundkörpers Triafulvalen (1) zu synthetisieren (Schema I). Einerseits könnte versucht werden, die PhS-Gruppen von $\mathbf{3 a}$ in gute Abgangsgruppen umzuwandeln, was nach unseren früheren Erfahrungen mit analogen Triafulven-Vorstufen nicht so einfach ist [6]. Andererseits wäre die Einführung zweier sich bei der geplanten Elimination unterstützender Abgangsgruppen attraktiv. Eine kürzlich erschienene

\section{Schema 1}

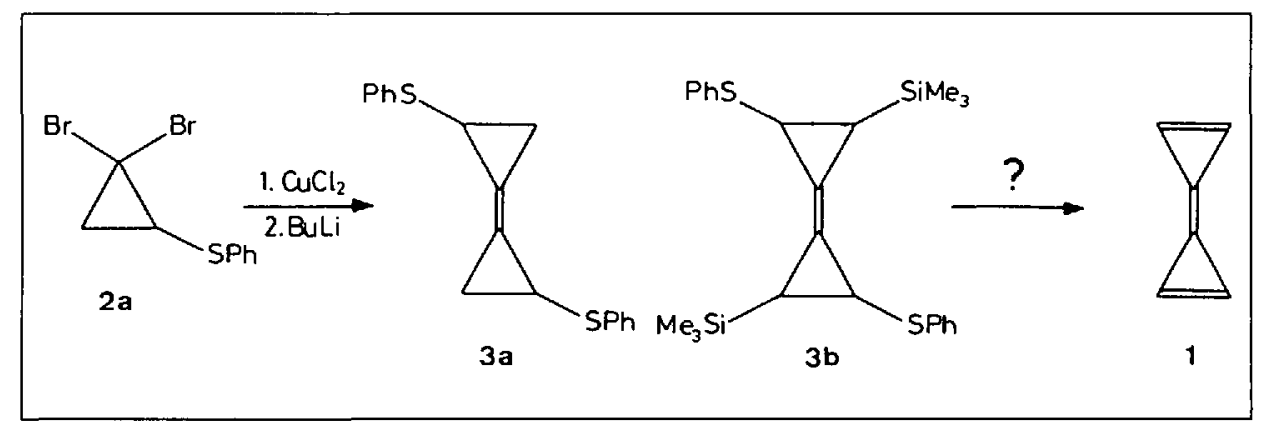


allem hohe Konzentrationen an $\mathbf{2}$ günstig sind. Ebenso ist die Diastereoselektivität des Kupplungsschrittes klein, indem laut ${ }^{1} \mathrm{H}-N M R-U n t e r s u c h u n g e n$ alle vier diastereoisimeren 'Carben-Dimere' 3b gebildet werden [13]. Immerhin wird ein Stereoisomer 3b bevorzugt gebildet und lässt sich mittels HPLC und Kristallisation rein darstellen.

Die spektroskopischen Daten des isolierten 'Carben-Dimers' 3b bestätigen bis auf die gegenseitige Anordnung der Substituenten dessen Struktur. So liegen im ${ }^{13} \mathrm{C}$-NMR-Spektrum $\left(\mathrm{CDCl}_{3}\right)$ alle Dreiring-C-Atome bei kleiner Frequenz $(\mathrm{C}(1)$ : 115,$4 ; C(2): 20,1 ; C(3): 20,3$ ppm), und im ${ }^{1} \mathrm{H}$-NMR-Spektrum erzeugen die DreiringProtonen aufgrund der $5 \mathrm{~J}$-Fernkopplungen über die zentrale $(\mathrm{C}=\mathrm{C})$-Bindung ein $A A^{\prime} X X^{\prime}$-System. Ebenso passen im MS die Masse des Molekülions wie auch die Hauptfragmentierungen zur vorgeschlagenen Struktur.

Die Oxidation $\mathbf{2 b} \rightarrow \mathbf{3 c}$ lässt sich mit guten Ausbeuten (rund 60\%) mittels $m$ Chloroperbenzoesäure realisieren (Schema 2). Alle spektroskopischen Daten belegen die Struktur des Disulfons 3c. Auch dessen 'H-NMR-Spektrum zeigt das auffällige $A A^{\prime} X X^{\prime}$ '-System der Dreiring-Protonen, deren Aufspaltungsmuster durch $5 J$-Kopplungen über die zentrale $(\mathrm{C}=\mathrm{C})$ Bindung kompliziert wird.

Unsere Ergebnisse zeigen, dass die kürzlich von uns gefundene Cu ${ }^{\text {II }}$-katalysierte 'Carben-Dimerisierung' erfolgreich auf die Synthese aussichtsreicher Triafulvalen-Vorstufen des Typs $\mathbf{3 b}$ und $\mathbf{3 c}$ angewandt werden kann. Nachteilig ist einzig die überraschend schlechte Ausbeute des Dimerisierungsschrittes $\mathbf{2 b} \rightarrow \mathbf{3 b}$, während die schlechte Diastereoselektivität im Hinblick auf die Synthese von 1 nicht stört. Bei Versuchen zur Fluorid-induzierten Eliminierung $\mathbf{3 c} \rightarrow \mathbf{1}$ konnte bisher in Gegenwart von Cyclopentadien kein DielsAlder-Addukt von $\mathbf{1}$ isoliert werden; doch wurden die Experimente durch die geringen verfügbaren Mengen an $\mathbf{3 b}$ behindert und werden fortgesetzt. Schliesslich ist von Interesse, dass bei der Behandlung von 1,1-Dibromo-2-(phenylsulfonyl)-3(trimethylsilyl)cyclopropan (2c) mit $\mathrm{Bu}_{4} \mathrm{NF}$ bei $-20^{\circ}$ in Gegenwart von Cyclopentadien mit $72 \%$ Ausbeute der kristalline Tricyclus 5 isoliert werden kann (Sche$m a$ 2). Damit wird belegt, dass in $2 \mathrm{c}$ das $\mathrm{Br}$-Atom die bessere Abgangsgruppe ist als die $\mathrm{PhS}$-Gruppe.

\section{Röntgenstrukturanalyse des bevor- zugt gebildeten Stereoisomers $3 \mathrm{~b}$}

Zur Kristallzüchtung wurde eine gesättigte Lsg. von 3b in Hexan/ $/ \mathrm{Et}_{2} \mathrm{O} 99: 1$ während $7 \mathrm{~d}$ auf $3^{\circ}$ gekühlt. So konnten farblose Kristalle mit Schmp. 139-140 gewonnen werden.

Schema 2

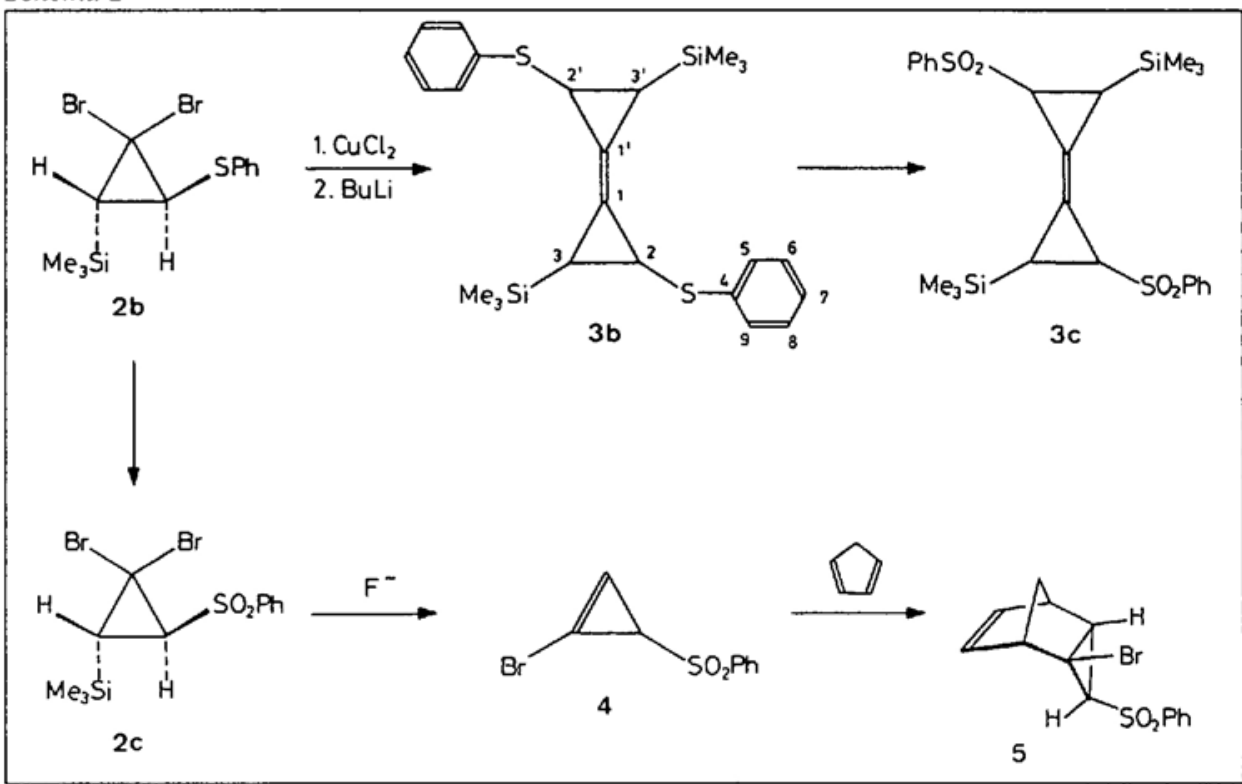

Tabelle. Bindungslängen $[\AA ̊]$ und Bindungswinkel $\left[^{\circ}\right]$ mit Standardabweichungen von $\mathbf{3 b}$

\begin{tabular}{llll} 
Bindung & Bindungslänge & Bindungen $\left.^{3}\right)$ & Bindungswinkel \\
\hline $\mathrm{C}(1)-\mathrm{C}\left(1^{2}\right)$ & $1,31(1)$ & $\mathrm{C}\left(1^{1}\right)-\mathrm{C}(1)-\mathrm{C}(2)$ & $146,8(1,5)$ \\
$\mathrm{C}(1)-\mathrm{C}(2)$ & $1,46(1)$ & $\mathrm{C}\left(1^{1}\right)-\mathrm{C}(1)-\mathrm{C}(3)$ & $148,0(1,7)$ \\
$\mathrm{C}(1)-\mathrm{C}(3)$ & $1,48(1)$ & $\mathrm{C}(2)-\mathrm{C}(1)-\mathrm{C}(3)$ & $64,9(0,9)$ \\
$\mathrm{C}(2)-\mathrm{C}(3)$ & $1,58(1)$ & $\mathrm{C}(1)-\mathrm{C}(2)-\mathrm{C}(3)$ & $58,2(0,7)$ \\
$\mathrm{C}(2)-\mathrm{S}$ & $1,80(1)$ & $\mathrm{C}(1)-\mathrm{C}(3)-\mathrm{C}(2)$ & $56,9(0,8)$ \\
$\mathrm{C}(3)-\mathrm{Si}$ & $1,86(1)$ & $\mathrm{C}(4)-\mathrm{S}-\mathrm{C}(2)$ & $102,3(0,4)$ \\
$\mathrm{C}(4)-\mathrm{S}$ & $1,79(1)$ & $\mathrm{C}(5)-\mathrm{C}(4)-\mathrm{C}(9)$ & $121,2(0,8)$ \\
$\mathrm{C}(4)-\mathrm{C}(5)$ & $1,38(2)$ & $\mathrm{C}(4)-\mathrm{C}(5)-\mathrm{C}(6)$ & $120,2(1,1)$ \\
$\mathrm{C}(4)-\mathrm{C}(9)$ & $1,36(2)$ & $\mathrm{C}(5)-\mathrm{C}(6)-\mathrm{C}(7)$ & $119,2(1,0)$ \\
$\mathrm{C}(5)-\mathrm{C}(6)$ & $1,39(2)$ & $\mathrm{C}(6)-\mathrm{C}(7)-\mathrm{C}(8)$ & $120,4(0,8)$ \\
$\mathrm{C}(6)-\mathrm{C}(7)$ & $1,38(2)$ & $\mathrm{C}(7)-\mathrm{C}(8)-\mathrm{C}(9)$ & $120,1(1,1)$ \\
$\mathrm{C} 7)-\mathrm{C}(8)$ & $1,38(2)$ & $\mathrm{C}(4)-\mathrm{C}(9)-\mathrm{C}(8)$ & $118,9(1,0)$ \\
$\mathrm{C}(8)-\mathrm{C}(9)$ & $1,41(2)$ &
\end{tabular}

a) Numerierung vgl. Schema 2.

Für die Röntgenstrukturanalyse wurde ein Kristall von ungefähr $0,3 \times 0,3 \times 0,3 \mathrm{~mm}$ präpariert. Erste Röntgenaufnahmen mit einer Weissenberg-Kamera und $\mathrm{Cu} K_{\alpha}$-Strahlung zeigten dass die Kristalle orthorhombisch sind mit der Raumgruppe Pbcn. Die Bestimmung der Gitterparameter $(a=10,895(5) ; b=10,697(5) ; c=$ $21,97(1) \AA$ ) und die Intensitätsmessungen erfolgten mit einem Nonius CAD-4-Diffraktometer und Mo $K \alpha$-Strahlung bei RT. DieZellparameterwurden nach der Methode der kleinsten Fehlerquadrate berechnet unter Berücksichtigung von 20 genau gemessenen Reflexen im Bereich $7,8^{\circ}<$ $2 \theta<13,8^{\circ}$. Anschliessend wurden die Intensitäten von allen unabhängigen Reflexen im Bereich $1^{\circ}<2 \theta<50^{\circ}$ gemessen. Jeweils nach 400 gemes senen Reflexen wurde die Orientierung des Kristalls überprüft und immer nach $3,8 \mathrm{~h}$ wurde die Intensität des (121)-Reflexes nachgemessen. Die gesamte Messung dauerte $\mathrm{ca} .48$ h. Insgesamt wurden 2595 Reflexe gemessen, davon 2248 unabhängige, von denen 737 als nichtbeobachte vermerkt wurden. Die Intensitäten wurden für Lorentz- und Polarisationseffekte korrigiert. Die Kristallstruktur konnte mit Hilfe der direkten
Phasenbestimmung ermittelt werden. Die Positionen der $\mathrm{H}$-Atome wurden aufgrund der Bindungsgeometrie berechnet. Die Struktur wurde nach der Methode der kleinsten Fehlerquadrate verfeinert. Für die C-, S- und Si-Atome wurden anisotrope Temperaturfaktoren verwendet. Der abschliessende Gütefaktor beträgt $R=0,13$ für die 1151 beobachteten Reflexe.

Die wichtigsten Daten der Molekülstruktur von $\mathbf{3 b}$ sind in der Tabelle und der Figur zusammengefasst. Wie erwartet liegen die C-Atome der Dreiringe in der Ebene der zentralen $(C=C)$ Bindung, welche mit 1,31 $\AA$ recht kurz ist. Typische Bindungslängen erhält man auch für die Dreiring-Bindungen. Dabei fällt auf, dass C(2) $\mathrm{C}(3)$ mit $1,58 \AA$ deutlich länger ist als die anderen Dreiring-Bindungen, ebenso dass der gegenüberliegende Dreiring-Winkel $C(2)-C(1)-C(3) \mathrm{mit}$ rund $65^{\circ}$ deutlich grösser ist als die andern Dreiring-Winkel von $57^{\circ}$ bzw. $58^{\circ}$. Vor allem zeigen die Röntgenstrukturdaten der Figur, dass die isolierte Verbindung eine trans-anti-Konfiguration aufweist, indem die beiden $\mathrm{PhS}-$ Gruppen (wie auch die beiden $\mathrm{Me}_{3} \mathrm{Si}$-Gruppen) bezüglich $\operatorname{der}(\mathrm{C}=\mathrm{C})$-Bindungsebene je in anti-Anordnung 


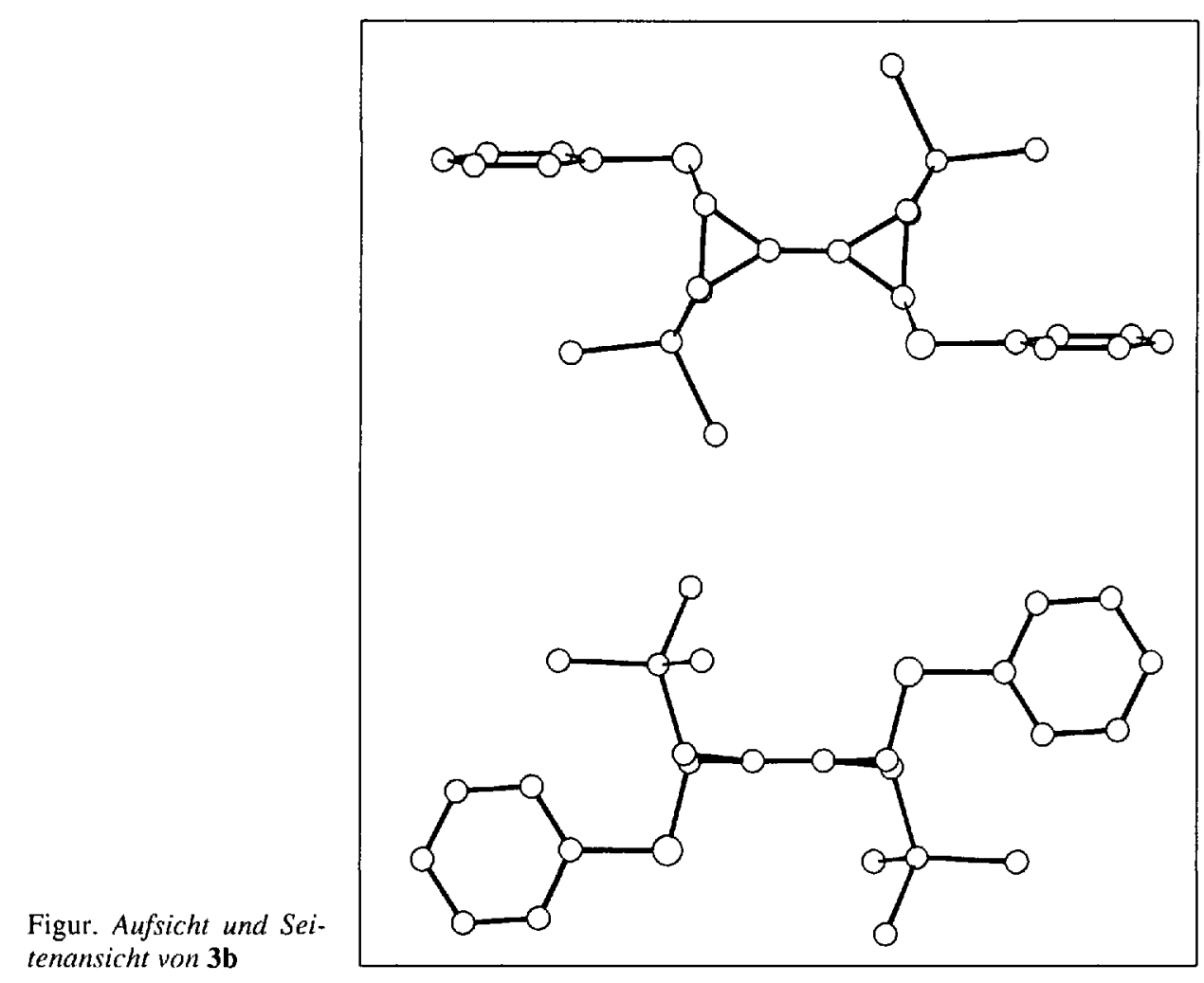

vorliegen. Offensichtlich ist bei der $\mathrm{Cu}^{\mathrm{Il}}$-katalysierten 'Carben-Dimerisierung' $\mathbf{2 b} \rightarrow \mathbf{3 b}$ das Diastereoisomere mit der energetisch günstigsten Konfiguration bevorzugt gebildet worden.

Die Autoren danken dem Schweizerischen Nationalfonds zur Förderung der wissenschaftlichen Forschung (Projekt Nr. 20-31217.91) für die Unterstützung der Arbeit.
Chimia 1991, 45, 382.

[4] S. You, M. Neuenschwander, Chimia 1992, $46,377$.

[5] A. Escher, W. Rutsch, M. Neuenschwander, Helv. Chim. Acta 1986, 69, 1644. Zur $\mathrm{CuCl}_{2}$-induzierten Kupplung des aus Bi(cyclopentadienyl)methan erhaltenen Dianions vgl. K. Hafner, G.F. Thiele, $J$. Am. Chem. Soc. 1985, I07, 5526.

[6] A. Weber, U. Stämpfli, M. Neuenschwander, Helv. Chim. Acla 1989, 72, 29.

[7] W.E. Billups, M.M. Haley, J. Am. Chem. Soc. 1991, 113, 5084.

[8] M. Makosza, in 'Modern Synthetic Methods 1976', Ed. R. Scheffold, Sauerländer, Aarau, 1976.

[9] W. von E. Doering, A. Hoffmann, J. Am Chem. Soc. 1954, 76, 6162.

[10] D. Seyferth, J.M. Burlitch, R. Minasz, J. Yick-Pui Mui, H. Simmons, A. Treiber, S. Dowd, J. Am. Chem. Soc. 1965, 87, 4259.

[11] Die Anordnung der Substituenten der Protonierungsprodukte geht aus den $1 \mathrm{H}-\mathrm{NMR}$ Spektren hervor und wird durch NOE-Experimente bestätigt.

[12] 'Thermodynamische Kontrolle' heisst: Zunächst wird $2 \mathrm{~b}$ bei $-95^{\circ}$ mit BuLi ungesetzt, dann zur Äquilibrierung während 30 min bei $-95^{\circ}$ gerührt und erst dann bei $-95^{\circ}$ mit $\mathrm{CuCl}_{2}$ versetzt. 'Kinetische Kontrolle' heisst: $2 \mathrm{~b}$ wird bei $-95^{\circ} \mathrm{mit} \mathrm{CuCl}_{2}$ versetzt, anschliessend tropft man bei $-95^{\circ}$ allmählich BuLi zu. Auf diese Weise wird das primär gebildete Carbenoid rasch umgesetzt.

1] Kurzmitteilung: 10. Mitteilung über Kupp lungsreaktionen. 9. Mitteilung vgl. [4]

[2] Teil der Diplomarbeit von Ch. L., Universität Bern, 1991.

[3] M. Borer, T. Loosli, M. Neuenschwander,
Chimia 46 (1992) 382-386

(C) Neue Schweizerische Chemische Gesellschaft ISSN $0009-4293$

\title{
Triafulven- und Calicen- Vorstufen für retro-Diels-Alder Reaktionen [1]
}

\author{
Michel Mühlebach [2] und Markus Neuenschwander*
}

Abstract. An attractive concept for the synthesis of triafulvene (1) and calicene (2) by retro-Diels-Alder reaction (RDA reaction) of precursors 3 and 4 is presented. Precursors 3 and 4 are easily available in three steps starting with dibenzobarrelene (5). First thermal-fragmentation experiments are summarized.

\section{Einleitung}

Seit rund 25 Jahren wird in verschiedenen Laboratorien versucht, die einfachsten Vertreter der Klasse der Fulvene und Fulvalene, Triafulven (1) und Pentatriafulvalen $(2$, Calicen) zu isolieren. Zahlreiche sterisch und elektronisch stabilisierte
Triafulvene und Calicene wurden synthetisiert, jedoch konnte der Grundkörper 1 erst vor wenigen Jahren spektroskopisch nachgewiesen werden [3][4], während Calicen (2) bis heute unbekannt ist. Die Schwierigkeiten bei der Synthese von 1 und 2 sind nicht nur auf ihre geringe thermische Stabilität zurückzuführen. Sie be- ruhen auch darauf, dass sich viele Verfahren zur Herstellung substituierter Triafulvene und Calicene nicht auf die Grundkörper übertragen lassen. So ergibt das vermutlich beste Verfahren, nämlich die Kupplung von Cyclopentadienid mit substituierten Cyclopropenylium-Kationen, wohl Calicene mit potentiellen Abgangsgruppen am Dreiring, doch führt die Abspaltung dieser Substituenten unter Dimerisierung zur Bildung von Bicalicen [5].

Ein grundsätzlich anderer SynthesePlan für 1 und 2 geht von den bisher unbekannten retro-Diels-Alder-Vorstufen des Typs 3 und 4 (Schema l) aus, welche möglicherweise ausgehend von Dibenzobarrelen (5) zugänglich sein könnten. Er basiert auf der plausiblen Annahme, dass die Energie-Bilanz der Retro-DielsAlder Reaktion (RDA-Reaktion) von 3 und 4 dadurch akzeptabel ausfällt, dass

*Korrespondenz: Prof. Dr. M. Neuenschwander Institut für organische Chemie

Universität Bern

Freiestrasse 3

$\mathrm{CH}-3012 \mathrm{Bem}$ 